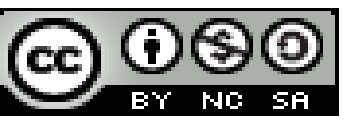

Ciencias Sociales y políticas

Artículo de investigación

\title{
La violencia de género y el derecho penal
}

\author{
Gender violence and criminal law
}

Violência de gênero e direito penal

\author{
Harold S. Quiñonez-Francis I \\ hasqui@hotmail.com
}

\author{
Magdalena J. Zambrano Guerrero II \\ magdalenazz@gmail.com \\ Máximo Prado Solís III \\ máximo.prados@utelvt.edu.ec
}

Recibido: 30 de enero de 2017 * Corregido: 20 de febrero de $2017 *$ Aceptado: 20 junio de 2017

I. Magister En Educación Mención En Psicodidáctica, Especialista en Derecho Procesal Penal, Magister en Administración de Empresas, Diplomado Superior en Docencia Universitaria, Diplomado Superior en Gestión Integral de Riesgos Y Desastres, Abogado de los Tribunales y Juzgados de la República del Ecuador, Docente de la Universidad Técnica Luis Vargas Torres de Esmeraldas, Esmeraldas, Ecuador.

II. Abogada de los Juzgados y Tribunales de la Republica, Magister en Gerencia Educativa, Docente de la Universidad Laica Eloy Alfaro de Manabí, Manta, Ecuador.

III. Master en Ciencias Especialidad Docencia Universitaria, Doctor en Ciencias de la Educación Mención Investigación y Planificación Educativa, Docente de la Facultad de Ciencias Sociales y Estudios del Desarrollo, Carrera de Sociología, Docente de la Universidad Luis Vargas Torres de Esmeraldas, Esmeraldas, Ecuador. 


\title{
Resumen
}

La violencia de género es un tipo de violencia física o psicológica ejercida contra cualquier persona o grupo de personas sobre la base de su sexo o género que impacta de manera negativa en su identidad y bienestar social, físico o psicológico. De acuerdo con la Organización de las Naciones Unidas, el término se utiliza «para distinguir la violencia común de aquella que se dirige a individuos o grupos sobre la base de su género». Se realizó una revisión bibliografía acerca de violencia de género cuyas consecuencias pueden trascender al ámbito del Derecho Penal, por tanto, son objeto de estudio de la criminología en su afán de prevenirlos.

Palabras clave: Violencia de género; prevención; derecho penal.

\begin{abstract}
Gender violence is a type of physical or psychological violence against any person or group of people based on their sex or gender that negatively impacts their identity and social, physical or psychological well-being. According to the United Nations Organization, the term is used "to distinguish common violence from that directed at individuals or groups on the basis of their gender." A bibliography review was made about gender violence whose consequences can transcend the scope of Criminal Law, therefore, they are the object of study of criminology in its eagerness to prevent them.
\end{abstract}

Key words: Gender violence; prevention; criminal law. 


\section{Introducción.}

Los derechos humanos, considerados como un conjunto de pautas éticas con proyección jurídica, surgen de la necesidad de todos los individuos de contar con las condiciones esenciales para una vida digna, y han sido producto de un largo proceso de construcción y cambio a lo largo de los dos últimos siglos. Aunque no se puede establecer una secuencia histórica lineal en la identificación de los derechos, sí se analizan los debates realizados en los organismos internacionales, de acuerdo a la historicidad con que ocurrieron. Se considera que los derechos civiles y políticos corresponden a la "primera generación"; los sociales, económicos y culturales, a la "segunda generación", y los derechos a la paz, al desarrollo y a un medio ambiente sano, a la "tercera generación", en tanto que la "cuarta generación" está representada por los derechos de los pueblos. (Rico N . 1996)

Hablar sobre violencia de género (Morrillas Cuevas L. 2002) propone situarnos ante un problema de una extraordinaria magnitud que abarca múltiples perpespectiva y en consecuencia requiere de respuestas también pluridisciplinarias. Su incidencia social es evidente y el esfuerzo solidario por combatirla cada vez más intenso y participativo, aunque todavía insuficiente. Durante siglos esta clase de violencia parecía enraizada en las más profundas tradiciones del dominio masculino, sintiéndose incluso como algo asumido dentro del entorno familiar o, al menos, como de solución interna en el ámbito doméstico, generalmente con el silencio de la mujer. La violencia como impotente manifestación de la desigualdad entre hombre y mujer es una vieja y decrepita formula a la que han recurrido y todavía recurren determinados varones para mantenerse en uno privilegio absolutamente rechazable a los que difícilmente podrían acceder de otra manera, como no sea a través de ciertos componentes religiosos que sobre un más que dudoso fundamentos de 
tradiciones y reglas claramente conculcadoras de los más esenciales derechos humanos pretenden reducir a la mujer a un mero objeto al servicio del hombre .

El Derecho Penal es la importante rama del derecho encargada de establecer todo un compendio de penas y castigos para imponerlos a quien haya cometido un delito, el cual amerite una condena por los actos cometidos. El Derecho Penal comprende una serie de leyes jurídicas con poder para privar de libertades y velar por el cumplimiento de las sanciones impuestas. El Derecho Penal dicta todo tipo de sentencia ante situaciones que puedan alterar de manera negativa el orden de la vida en la sociedad, si una persona infringe en el código civil y viola los estatutos morales impuestos por la ética y la moral del medio ambiente que lo rodea, se verá obligado a pagar por sus infracciones. (Definición de Derecho Penal. 2015).

El objetivo clave del derecho penal es proteger a la sociedad de las malas costumbres, fue ideado con el precepto de dejar en claro que el estado y las instituciones deben permanecer firmes a la corrupción y la maldad, fundando así sentimientos de libertad y buen vivir entre los ciudadanos. (Definición de Derecho Penal. 2015.)

Desarrollo.

La violencia de género en América Latina.

A nivel mundial parece que en nuestro entorno cultural el problema se afronta desde perspectivas distintas y más comprometidas con los parámetros de igualdad, solidaridad y respeto.

En América Latina y el Caribe, hasta los años noventa, la violencia contra las mujeres, principalmente, la acaecida en el ámbito familiar, era considerada un asunto privado en el cual el Estado no debía intervenir. Por otro lado, poco se conocía sobre la magnitud del problema, de 
manera que se tendía a asumir que la violencia hacia la población femenina ocurría de forma aislada, y no se la concebía como un problema social y de política pública. Esta concepción se expresaba en la invisibilidad de la violencia de género hacia las mujeres, tanto en el ámbito legislativo, como en el ejecutivo y en el judicial, como también en otros sectores de la sociedad.( Quintana Zurita Y et al 2014)

En este mismo orden y dirección, América Latina es hoy en día la región más insegura del mundo, al igual que la más desigual. La inequidad y la exclusión se traducen en altos niveles de violencia que afectan principalmente a mujeres, jóvenes, niños y poblaciones indígenas. El ejercicio de esa violencia es multicausal y multidimensional. La violencia es producto del impacto psicológico y pérdida del tejido social causado por los diversos conflictos armados vividos en la región; de la debilidad institucional y de la ausencia de políticas públicas incluyentes y equitativas. La proliferación de armas de fuego y el fácil acceso a las mismas exacerban la conflictividad e incrementan la letalidad. Desde una perspectiva de género, en la última década surgen dos fenómenos asociados a esa violencia: el feminicidio y la construcción de la masculinidad en las pandillas juveniles. La solución debe plantearse desde una perspectiva multidimensional, basada en la prevención y en las políticas de equidad e inclusión enfocadas en erradicar la violencia intrafamiliar y la proliferación de armas de fuego en la región. (De León Escribano C R . 2008)

Según Ibáñez D B (2017), catorce países latinoamericanos se encuentran entre los veinticinco países del planeta con una mayor incidencia de feminicidios. Otro estudio, realizado por (Geneva Smalls 2012), el Salvador (12 mujeres asesinadas por cada 100.000 habitantes femeninas), Guatemala (9,7 mujeres asesinadas por 100.000 habitantes del sexo femenino), Honduras (7 mujeres 
asesinadas por 100.000 habitantes femeninas), Colombia y Bolivia (alrededor de 6 mujeres asesinadas por cada 100.000 mujeres), son cinco de los doce países más violentos del mundo.

En el ecuador la violencia de género ha sido estudiada en numerosas contribuciones, sobre todo en los últimos años. Pero, a pesar de toda esta pléyade de contribuciones, faltan estudios que “capturen las experiencias subjetivas de las mujeres sobre la violencia”, por cuanto la investigación en este ámbito se basa fundamentalmente en patrones cuantificadores.( Ibáñez, D B. 2017)

Ecuador es un país que presenta una grave situación en cuanto a la violencia de género; según cifras oficiales, seis de cada diez mujeres reconocen haber padecido malos tratos, si bien muchas de las mujeres que sufren violencia no llegan a denunciar al agresor. En las últimas tres décadas, hubo importantes esfuerzos hacia la erradicación de la violencia de género en Ecuador. .( Ibáñez, D B. 2017)

- 1979. Se crea la "Oficina Nacional de la Mujer" en el Ministerio de Bienestar Social

- 1980. Se inauguran las de Casas de Acogida o Refugios para las víctimas en Quito, Guayaquil, Cuenca, Sucumbíos y Orellana.

- 1994. Ecuador firma la Convención Interamericana para Prevenir, Sancionar y Erradicar la Violencia contra la Mujer.

- 2007. Rafael Correa rubrica el Decreto Número 620: se declara como "política de Estado" la erradicación de la violencia.

- 2014. El Código Orgánico Integral Penal define la violencia de género como un fenómeno de carácter físico, psicológico y sexual.

La violencia de género: entre el concepto jurídico y la realidad social.

La preocupación social acerca de la violencia contra las mujeres no se concentra en ellas y en las razones que explican su victimización sino que se traslada a la familia y a sus miembros en tanto que víctimas propicias de la violencia masculina a consecuencia de una "natural" posición de 
inferioridad que es compartida por la mujer. No es de extrañar que ese planteamiento acabe favoreciendo la permisividad social de un maltrato cuyas causas se sitúan en una (asimismo) “natural” posición de superioridad del varón, de su autoridad -paterna, marital...- en tanto que garante del orden familiar. Son patrones culturales tan enraizados en la sociedad que han terminado por normalizarse, por considerarse "naturales"... De ahí también que el problema del maltrato permanezca en la privacidad, como un secreto, un tabú que no debe salir del ámbito doméstico y que el propio grupo debe resolver sin la intervención de terceros ajenos al conflicto, fomentándose así, como con razón se afirma, "uno de los prejuicios culturales que en mayor medida han obstaculizado la persecución de la violencia de género, que sigue siendo en la actualidad para muchos "un delito invisible. . ( La violencia de género: entre el concepto jurídico y la realidad social. 2004).

Esa confusión de etiquetas, a veces interesada, entre violencia de género y violencia doméstica, contribuye a perpetuar la probada resistencia social a reconocer que el maltrato a la mujer no es una forma más de violencia, que no es circunstancial ni neutra sino instrumental y útil en aras de mantener un determinado orden de valores estructuralmente discriminatorio para la mujer. Una visión de la realidad que sólo es posible alcanzarla desde una perspectiva de género que, a duras penas, consigue imponerse en la sociedad y, desde luego, en el Derecho que, en buena medida, no hace sino reproducir el discurso dominante Es significativo, por ejemplo, que la mayor parte de las declaraciones que se manifiestan interesadas por combatir la violencia de género recojan entre sus recomendaciones la de sensibilizar a la sociedad para que tome conciencia de la gravedad del problema y cambie su actitud hacia la violencia que se ejerce contra las mujeres. (La violencia de género: entre el concepto jurídico y la realidad social. 2004). 
Es evidente entonces que la perspectiva de la mujer debe estar necesariamente presente en el debate social y jurídico que busca soluciones al problema. Son sus bienes más relevantes su vida, su integridad, su libertad, su dignidad y no los de la familia en su conjunto los que están en juego, de modo que su defensa deben organizarse sobre la base de sus intereses personales. . (La violencia de género: entre el concepto jurídico y la realidad social. 2004).

El recurso a la protección del Estado, en muchos casos imprescindible, debiera ser altamente selectivo y garantizar a la mujer amplias cotas de libertad. Su perspectiva debe ser esencial a la hora de enfrentarse a la violencia que la somete. ( La violencia de género: entre el concepto jurídico y la realidad social. 2004).

La llamada a la autonomía personal de la mujer como clave para la solución de los conflictos que le atañen no es nueva para el feminismo. En sede penal resulta esclarecedora la polémica que surgió alrededor de los años ochenta del pasado siglo en Italia con motivo del proceso de reforma de los delitos contra la libertad sexual. La apuesta indiferenciada por la vía penal que propugnaba uno de los sectores feministas en pugna, se vio superada por el enfoque diferenciador de quienes defendían una persecución selectiva de esas agresiones en función de las necesidades particulares de sus víctimas reconociéndoles el derecho de renunciar a la acción penal. En este caso, se entendió con buen sentido que la perseguibilidad a instancia de parte dejaba un espacio irrenunciable a la mujer para decidir libremente conforme a sus intereses reales. ( La violencia de género: entre el concepto jurídico y la realidad social. 2004).

Los resultados de un proyecto en el que se generó casi tres mil encuestas a un grupo referente: los estudiantes universitarios. A grandes rasgos, se percibió un desconocimiento sobre 
todo entre los hombres de la complejidad debida al fenómeno de la violencia, así como cierta tolerancia hacia este tipo de epidemia social.( Ibáñez, D B. 2017)

\section{El derecho penal frente a la violencia de género}

Los estudios de género tienen poco que ver con el positivismo jurídico. Mientras el positivismo es el hijo predilecto de la modernidad, el feminismo se ha concentrado en destruir la presunción de igualdad y universalidad. (Zaikoski Biscay D. 2012).

Este paradigma supone un saber jurídico naturalizado, dogmatizado y restringido a la letra de la ley. Por el contrario, si se cree que lo jurídico es contingente, producto de luchas sociales, tan inestable y social como cualquier otro conocimiento, entonces sí puede entablarse un diálogo fecundo entre género y derecho para revertir las violencias sociales. (Zaikoski Biscay D. 2012).

El gran debate político criminal es si realmente hace falta que el derecho penal intervenga de alguna manera específica para proteger a las mujeres que se encuentran en situación de violencia o si, por el contrario, bastan los pos generales, los pos genéricos que existen en todas las legislaciones. Este debate se ha extendido por todos los países desde hace aproximadamente unos diez o quince años. (Laurenzo P. 2012)

Sobre todo (Laurenzo P. 2012), en los últimos diez años, han sido muy claras las exigencias de los movimientos de mujeres solicitando penas más elevadas para los maltratadores y también formas de cumplimiento más severas de las penas. 
El objetivo del legislador fue, desde el primer momento, buscar mecanismos específicos para combatir la violencia contra las mujeres en la pareja, o quizá abriéndolo en algo más.

La importancia que tiene este momento histórico, desde el punto de vista del cambio político criminal, es que como consecuencia de ese reconocimiento del derecho positivo, el concepto de violencia de género se incorpora también a la legislación penal.

Cómo prevenir la violencia de género.

La violencia de género es un tema que en la actualidad está siendo tratado de forma multidisciplinar desde distintos sectores para completar un proceso con un objetivo único; la promoción de la igualdad.

La Prevención Primaria cuya principal estrategia es la educativa, se dirige a grupos y colectivos; no se trata de una actuación individual. (Cómo prevenir la violencia de género. 2017)

Se orienta fundamentalmente a las generaciones jóvenes. Niños y niñas son educados en igualdad con la intención de que construyan unas pautas de comportamiento, creencias y actitudes que mejoren su empoderamiento e independencia personal y las actuales relaciones entre mujeres y hombres y que, por tanto, eviten la violencia de género. (Cómo prevenir la violencia de género. 2017)

Un criterio esencial para trabajar la prevención de la violencia de género, consiste en proporcionar información, ideas y propuestas para la actuación, incorporando valores que eviten la violencia y descalifiquen aquellos valores que la propicien. (Cómo prevenir la violencia de género. 2017) 
Notas. (Rico N. 1996)

1. Es importante destacar que América Latina y el Caribe es la primera, y hasta el momento la única, región del mundo en la que todos los países han ratificado la Convención sobre la eliminación de todas las formas de discriminación contra la mujer. Sin embargo, esto no significa que ya hayan adaptado su legislación a la Convención en todos los campos, que hayan implementado políticas y adoptado acciones positivas para eliminar la discriminación de facto, ni que cumplan con la obligación de informar al Comité para la Eliminación de la Discriminación en Contra de la Mujer (CEDAW).

2. Según el grupo de expertos sobre violencia contra la mujer de las Naciones Unidas, toda persona puede ser víctima de actos de violencia, pero el sexo es uno de los factores que aumentan significativamente su vulnerabilidad. Algunos de los elementos que permiten afirmar que existe violencia de género son los siguientes: a) la mayoría de los agresores son hombres, independientemente de que la víctima sea varón o mujer; b) la violencia afecta de distinta manera a los varones y las mujeres, debido a que los daños que sufren suelen estar determinados por su sexo; c) los agresores suelen estar motivados por consideraciones de género, como la necesidad de fortalecer el poder y los privilegios masculinos (Naciones Unidas, 1993a).

Se concluye que la creación legislativa de este tipo penal no se reduciría a un acto aislado del Estado, sino que sería el inicio de una política criminológica más amplia

\section{Referencias bibliográficas.}

Cómo prevenir la violencia de género [sitio web]. 2017 [consulta 25 octubre 2017]. Disponible en: http://www.divulgaciondinamica.es/blog/prevenir-violencia-genero/

Definición de Derecho Penal [sitio web]. 2015 [consulta 25 octubre 2017]. Disponible en: http://conceptodefinicion.de/derecho-penal/ 
DE LEÓN ESCRIBANO C R . 2008. Violencia y género en América Latina [consulta 25 octubre 2017]. Disponible en:https://dialnet.unirioja.es/servlet/articulo?codigo=2873321

GENEVA SMALLS ARMS SURVEY. 2012: Moving Targets. Cambridge: Cambridge University Press,

IBANEZ, D B 2017. La violencia de género en Ecuador: un estudio sobre los universitarios. Rev. Estud. Fem., 25(3) [consulta 25 octubre 2017], pp.1313-1327. ISSN 0104026X. Disponible en: <http://www.scielo.br/scielo.php?script=sci_arttext\&pid=S0104026X2017000301313\&lng=es\&nrm=iso>.http://dx.doi.org/10.1590/18069584.2017v25n3p1313.

La violencia de género: entre el concepto jurídico y la realidad social [sitio web]. 2004. Proyecto de investigación financiado por el ministerio de educación y ciencia " análisis de la lo. $1 / 2004$ de protección integral contra la violencia de las mujeres desde una perspectiva de género" (sej 2005 - 064 / juri). [consulta 25 octubre 2017]. Disponible en pmayobre.webs.uvigo.es/06/arch/profesorado/inma_valeije/violencia.doc

LAURENZO P. 2012. El Derecho Penal Frente a la Violencia de Género. R. EMERJ, Rio de Janeiro, v. 15, n. 57. p. 142-154,

MORRILLAS CUEVAS L. 2002. Valoración de la violencia de género desde la perspectiva del derecho Penal. Revista Electrónica Ciencias Penal y criminología. [consulta 25 octubre 2017]. Disponible en: criminet.ugr.es/recpc/recpc_04-09.pdf

QUINTANA ZURITA Y [et al.]. 2014. La violencia de género contra las mujeres. En el ecuador. [consulta 25 octubre 2017]. ISBN 978-9942-07-761-5 Disponible en . https://www.unicef.org/ecuador/Violencia_de_Gnero.pdf

RICO N. 1996. Violencia de género: un problema de derechos humanos [consulta 25 octubre 2017]. Disponible en: https://www.cepal.org/mujer/noticias/paginas/3/27403/violenciadegenero.pdf

ZAIKOSKI BISCAY D. 2012. La violencia familiar y prácticas de los operadores jurídicos del derecho penal: análisis de cinco casos de Santa Rosa, Argentina. Revista Punto Género No 2. ISSN 0719-0417 / 93 - 117 